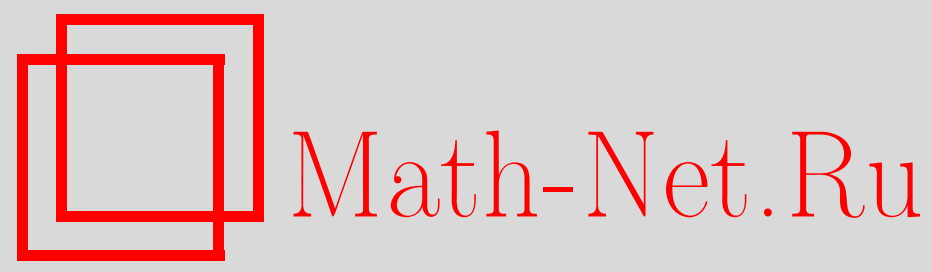

А. М. Зубков, Г. И. Ивченко, Ю. И. Медведев, Корни производящих функций и суммы целочисленных случайных величин, Матем. вопр. криптогр., 2020, том 11, выпуск 1, 2746

DOI: https://doi.org/10.4213/mvk313

Использование Общероссийского математического портала Math-Net.Ru подразумевает, что вы прочитали и согласны с пользовательским соглашением http://www.mathnet.ru/rus/agreement

Параметры загрузки:

IP: 35.173 .219 .149

26 апреля 2023 г., $15: 18: 46$

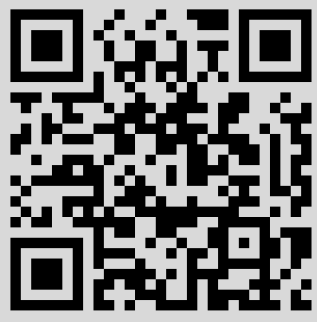


МАТЕМАТИЧЕСКИЕ ВОПРОСЫ КРИПТОГРАФИИ

2020 T. 11 № 1 C. 27-46

УДК $519.212 .2+519.115$

DOI https://doi.org/10.4213/mvk313

\title{
Корни производящих функций и суммы целочисленных случайных величин
}

\author{
А. М. Зубков ${ }^{1}$, Г. И. Ивченко ${ }^{2}$, Ю. И. Медведев ${ }^{2}$ \\ ${ }^{1}$ Математический институт им. В.А. Стеклова Российской \\ академии наук, Москва \\ ${ }^{2}$ Академия криптографии Российской Федеращии, Москва
}

Получено 29.IV.2019

Аннотация. Рассматриваются свойства корней производящих функций целочисленных ограниченных случайных величин и свойства сумм независимых случайных величин со значениями в множествах $\{0,1\}$ и $\{0,1,2\}$. Указаны условия сходимости распределений целочисленных ограниченных случайных величин к пуассоновскому и нормальному распределениям в терминах корней производящих функций.

Ключевые слова: целочисленные случайные величины, корни производящих функций, суммы независимых простейших случайных величин, предельные теоремы

The roots of generating functions and sums of integer-valued random variables
A. M. Zubkov ${ }^{1}$, G. I. Ivchenko ${ }^{2}$, Yu. I. Medvedev ${ }^{2}$
${ }^{1}$ Steklov Mathematical Institute of Russian Academy of Sciences, Moscow
${ }^{2}$ Academy of Cryptography of the Russian Federation, Moscow

\begin{abstract}
Properties of roots of generating functions of integer-valued bounded random variables and properties of sums of independent random variables with values in sets $\{0,1\}$ and $\{0,1,2\}$ are studied. Conditions of weak convergence of integer-valued bounded random variables to the Poisson and normal laws in terms of roots of generating functions are presented.
\end{abstract}

Keywords: integer-valued random variables, roots of probability generating functions, sums of independent simplest random variables, limit theorems 


\section{1. Введение. Постановка задачи и предварительные утверждения}

Пусть $\xi$ - целочисленная случайная величина (с. в.), принимающая значения $0,1, \ldots, n$, и $P(z)=\mathbf{E} z^{\xi}-$ ее производящая функция (пр. ф.):

$$
P(z)=p_{0}+p_{1} z+\ldots+p_{n} z^{n}, \quad p_{0}, p_{n}>0 .
$$

Множество (класс) таких случайных величин обозначим $\mathrm{M}_{n}$. В настоящей работе изучаются способы построения для любой случайной величины $\xi \in \mathrm{M}_{n}$ таких конечных линейных комбинаций случайных величин:

$$
\xi+\sum_{k \geqslant 1} d_{k} \zeta_{k} \quad \text { и } \sum_{m \geqslant 1} c_{m} \xi_{m}
$$

что распределения этих сумм совпадают, здесь коэффициенты $d_{k}$ и $c_{m}$ - натуральные числа, величины $\xi_{m}$ и $\zeta_{k}$ независимы, не зависят от $\xi$ и принадлежат классам $\mathrm{M}_{1}$ и $\mathrm{M}_{2}$.

Случайные величины из классов $\mathrm{M}_{1}$ и $\mathrm{M}_{2}$ будем называть далее простейшими.

Разложения типа (2) можно использовать, например, для оценивания моментов случайных величин. Важный частный случай, когда все корни производящей функции $P(z)$ действительные, хорошо изучен, и ему посвящено значительное число публикаций. Много подобных примеров можно найти в задачах вероятностной комбинаторики: теории случайных размещений, случайных подстановок, случайных разбиений конечных множеств и других моделей комбинаторных объектов (см., например, $[1,4,8,11]$. Ряд других примеров приводится ниже (см. раздел 5).

Значительно меньше изучен случай, когда не все корни действительные. В частности, случай, когда все корни пр. ф. $P(z)=\mathbf{E} z^{\xi}$ лежат в левой полуплоскости $\operatorname{Re} z \leqslant 0$, был рассмотрен (в кратком изложении и без доказательств) в [3]. В настоящей работе эти результаты существенно обобщаются, особенно в отношении нулей производящих функций.

Покажем, что одинаково распределенные суммы вида (2) с $d_{1}=$ $d_{2}=\ldots=0$ существуют лишь тогда, когда все корни многочлена $P(z)$ лежат в левой полуплоскости Re $z \leqslant 0$. В случаях же, когда многочлен $P(z)$ имеет корни и в правой полуплоскости, существуют одинаково распределенные суммы вида $(2)$ с ненулевыми $d_{m}$.

Случайную величину $\zeta=\sum_{k \geqslant 1} d_{k} \zeta_{k}$ (как и ее производящую функцию $\left.\mathbf{E} z^{\zeta}\right)$ будем называть компенсатором для $\xi$. 


\section{2. Свойства корней производящих функций}

Обозначим корни (нули) многочлена (1) через

$$
z_{k}=\rho_{k} e^{i \varphi_{k}}, \quad k=1, \ldots, n .
$$

Производящую функцию $P(z)$ можно представить в виде

$$
P(z)=\prod_{k=1}^{n} \frac{z-z_{k}}{1-z_{k}}=\prod_{k=1}^{n}\left(b_{k}+a_{k} z\right),
$$

где $a_{k}=\frac{1}{1-z_{k}}, \quad b_{k}=1-a_{k}=\frac{-z_{k}}{1-z_{k}}, \quad k=1, \ldots, n$.

Если все корни производящей функции лежат на отрицательной полуоси, то величины $a_{k}=p_{k}$ и $b_{k}=q_{k}$ можно рассматривать в качестве вероятностей бернуллиевского распределения, а $P(z)$ в этом случае представляет собой производящую функцию обобщенного биномиального распределения. Если корни могут принимать комплексные значения, то величины $a_{k}$ и $b_{k}$ являются комплексными аналогами параметров бернуллиевской схемы. Как будет далее, интересующие нас функционалы от этих параметров являются действительными величинами.

Отметим одно важное свойство величин $a_{k}$. Назовем величину

$$
A_{j}=\sum_{k=1}^{n} a_{k}^{j}
$$

$j$-м корневым функционалом пр. ф. $P(z), j=1,2, \ldots$

Значение этих величин определяется тем, что характеристическая функция, а следовательно, и моменты (обычный, центральный, факториальный и т. д.) рассматриваемой случайной величины однозначно выражаются через эти корневые функционалы. А именно:

$$
\mathbf{E} e^{i t \xi}=P\left(e^{i t}\right)=\exp \left\{\sum_{j=1}^{\infty} \frac{\left(1-e^{i t}\right)^{j}}{j} A_{j}\right\} .
$$

При этом все $A_{j}$ являются действительными величинами. Это следует из того, что факториальные семиинварианты

$$
\kappa_{[j]}=\operatorname{coef}_{z^{j} / j !} \omega(z),
$$

где

$$
\begin{gathered}
\omega(z)=\ln P(1+z) \\
=\ln \prod_{k=1}^{n}\left(1+a_{k} z\right)=\sum_{k=1}^{n} \sum_{j=1}^{\infty} \frac{(-1)^{j-1} a_{k}^{j}}{j} z^{j}=\sum_{j=1}^{\infty} \frac{(-1)^{j-1} A_{j}}{j} z^{j},
\end{gathered}
$$


выражаются через корневые функционалы:

$$
\kappa_{[j]}=(-1)^{j-1}(j-1) ! A_{j}, \quad j=1,2, \ldots
$$

Очевидно, что и любые функционалы вида

$$
A_{j, l}=\sum_{k=1}^{n} a_{k}^{j} b_{k}^{l}, \quad j, l=0,1,2, \ldots
$$

также являются действительными величинами и через них также можно выразить любые моменты. Так, например, для первых четырех обычных семиинвариантов

$$
\kappa_{m}=\operatorname{coef}_{\frac{{ }^{m}}{m !}} \ln P\left(e^{t}\right)=\sum_{j=1}^{m} S(m, j) \kappa_{[j]},
$$

где $S(m, j)$ - числа Стирлинга второго рода, соответствующие формулы имеют вид

$$
\begin{aligned}
& \kappa_{1}=\mathbf{E} \xi=A_{1,0}, \quad \kappa_{2}=\mathbf{D} \xi=A_{1,1}, \\
& \kappa_{3}=\mathbf{E}\left(\xi-A_{1}\right)^{3}=A_{1,1}-2 A_{2,1}, \quad \kappa_{4}=A_{1,1}-6 A_{2,2} .
\end{aligned}
$$

Приведем также несколько вспомогательных утверждений о корнях производящих функций.

Лемма 1. 1) $B$ секторе $0 \leqslant|\varphi|<\frac{\pi}{n}$ nр. $\oint . P(z)$ корней не имеет.

2) В секторе $0 \leqslant|\varphi|<\frac{\pi}{n-l}$ nр. $\oint . P(z)$ корней кратности $l+1$ не uмeеm.

3) Хотя бъ один корень $P(z)$ расположен в левой полуплоскости Re $z \leqslant 0$.

Доказательство. 1. Все слагаемые суммы

$$
\operatorname{Im} P\left(z_{k}\right)=\sum_{j=1}^{n} p_{j} \rho_{k}^{j} \sin \left(j \varphi_{k}\right)
$$

в секторе $0 \leqslant|\varphi|<\frac{\pi}{n}$ неотрицательны, а последнее слагаемое больше нуля.

2. Корень пр. ф. $P(z)$ кратности $l+1$ остается корнем у $l$-й производной $P^{(l)}(z)$, которая является многочленом степени $n-l$, значит, к нему применимо утверждение 1 леммы. 
3. Утверждение 3 следует из формулы Виета

$$
\sum_{k=1}^{n} \operatorname{Re} z_{k}=-\frac{p_{n-1}}{p_{n}}
$$

Лемма 2. Если один из корней пр. $\varnothing . P(z)$ лежит на границе сектора $0 \leqslant|\varphi| \leqslant \frac{\pi}{n}\left(\right.$ и имеет вид $\left.\tilde{z}=\tilde{\rho} e^{i \frac{\pi}{n}}\right)$, то он однозначно определяет $P(z)$ :

$$
P(z)=p_{0}+p_{n} z^{n}, \quad p_{0}=\frac{\tilde{\rho}^{n}}{1+\tilde{\rho}^{n}}, \quad p_{n}=\frac{1}{1+\tilde{\rho}^{n}} .
$$

В этом случае $\tilde{\rho}=\left(\frac{p_{0}}{p_{n}}\right)^{1 / n}$, и все корни находятся на окружности paдиуса $\tilde{\rho}: z_{k}=\tilde{\rho} e^{i \pi(1+2 k) / n}, k=0,1, \ldots, n-1$.

Доказательство. Из (6) следует, что соотношение

$$
\operatorname{Im} P(\tilde{z})=0
$$

возможно лишь в случае, когда $p_{1}=p_{2}=\ldots=p_{n-1}=0$, а из условия

$$
\operatorname{Re} P(\tilde{z})=\sum_{j=0}^{n} p_{j} \tilde{\rho}^{j} \cos \left(j \frac{\pi}{n}\right)=0
$$

следует, что $p_{0}-p_{n} \tilde{\rho}^{n}=0$. Отсюда однозначно определяются как указанные в (7) значения коэффициентов, так и вид корней.

Следствие. В условиях леммы 2 справедливо представление

$$
\xi=n \xi_{1}, \quad \xi_{1} \in \mathrm{M}_{1} .
$$

Отметим, что (см. [14]) число корней производящей функции (1) в правой полуплоскости в секторе $|\operatorname{Arg} z| \leqslant \alpha(\alpha \in(0, \pi / 2))$ не превосходит $2 \alpha n / \pi$.

Лемма 3. С. в. является решетчатой с шагом $d>1$ тогда и только тогда, когда множество всех корней ее пр. $\oint$. $P(z)$, рассматриваемое как множество точек в z-плоскости, переходит в себя при повороте плоскости на угол $2 \pi / d$.

Доказательство достаточно очевидное. Заметим, что эта лемма аналог известной теоремы о периодичности характеристической функции случайной величины, решетчатой с шагом решетки $d>1$. 
Следствие. Если все корни пр. ф. лежат в левой полуплоскости и шаг решетки равен двум, то все корни лежат на мнимой оси.

Лемма 4 (Взаимно симметричные случайные величины). Пусть с. в. $\xi \in \mathrm{M}_{n}$ и корни ее пр. ф. $P(z)$ указаны в (3). Тогда с. в. $\xi^{\prime}=n-\xi \in \mathrm{M}_{n}$ и корни ее пр. ф. $Q(z)=z^{n} P\left(z^{-1}\right)=\prod_{k=1}^{n}\left(a_{k}+b_{k} z\right)$ суть $z_{k}^{\prime}=\rho_{k}^{-1} e^{-i \varphi_{k}}$, $k=1, \ldots, n$.

Доказательство очевидно.

Лемма 5 (Сопряженные с. в.). Пусть $\xi^{\prime}(a)$ - случайная величина, сопряжсенная случайной величине $\xi$ с параметром $a>0$ :

$$
\mathbf{P}\left\{\xi^{\prime}=k\right\}=c \mathbf{P}\{\xi=k\} a^{k}, \quad k=0,1,2, \ldots, n
$$

(с- нормирующая константа).

Тогда корни ее пр. $\phi . P_{\xi^{\prime}(a)}(z)=\frac{P(a z)}{P(a)}$ равны $z_{k}^{\prime}=\frac{\rho_{k}}{a} e^{i \varphi_{k}}, k=1, \ldots, n$, где $\rho_{k}$ и $\varphi_{k}-$ те же, что в (3).

Доказательство очевидно.

Лемма 6. Если все корни пр. $\oint . P(z)$ расположены на окружности радиуса a, mo

$$
p_{k}=p_{n-k} a^{n-2 k}, \quad k=0,1, \ldots, n .
$$

Доказательство. При условии леммы все корни пр. ф. $Q(z)=\frac{P(a z)}{P(a)}$ расположены на единичной окружности, и пр. ф. $z^{n} Q\left(z^{-1}\right)$ имеет те же корни. Следовательно, $Q(z)=z^{n} Q\left(z^{-1}\right)$. Приравнивая коэффициенты этих пр. ф., получаем утверждение.

\section{3. Построение компенсаторов для случайных величин из $\mathrm{M}_{n}$}

Перейдем теперь к вопросу о существовании представлений вида (2). Класс $\mathrm{M}_{1}$ - это класс бернуллиевских случайных величин. Значение этого класса в ситуациях, когда все корни производящей функции (1) действительны, хорошо известно (см. приводимые ниже примеры в разделе 5).

Класс $\mathrm{M}_{2}$ - это класс случайных величин с производящими функциями вида

$$
\mathbf{E} z^{\xi}=P\left(z, z_{1}\right)=p_{0}+p_{1} z+p_{2} z^{2}=p_{2}\left(z-z_{1}\right)\left(z-\bar{z}_{1}\right) .
$$


Очевидно, ее корни, $z_{1}=\rho e^{i \varphi}, z_{2}=\bar{z}_{1}$, действительны при $\varphi= \pm \pi$ и комплексно сопряжены в противном случае, при этом

$$
\rho=\left(p_{0} / p_{2}\right)^{1 / 2}, \quad \cos \varphi=-\frac{1}{2} \frac{p_{1}}{\sqrt{p_{0} p_{2}}} \leqslant 0
$$

(корни лежат в левой полуплоскости).

С другой стороны,

$$
p_{2}=\left(1-2 \rho \cos \varphi+\rho^{2}\right)^{-1}, \quad p_{1}=-2 \rho \cos \varphi \cdot p_{2}, \quad p_{0}=\rho^{2} p_{2} .
$$

Если $p_{1}=0\left(\Leftrightarrow \varphi=\frac{\pi}{2}\right)$, то корни расположены на мнимой оси, и соответствующая случайная величина представляет собой удвоенную бернуллиевскую величину.

Моменты случайной величины из $\mathrm{M}_{2}$ выражаются через корни ее производящей функции следующим образом:

$$
\begin{aligned}
& \mathbf{E} \xi^{k}=2 p_{2}\left(2^{k-1}-\rho \cos \varphi\right), \\
& \mathbf{D} \xi=2 p_{2}(2-\rho \cos \varphi)-4 p_{2}^{2}(1-\rho \cos \varphi)^{2} .
\end{aligned}
$$

Ниже нам понадобится простой алгебраический факт, проясняющий роль функций вида (8).

Лемма 7. Пусть

$$
h\left(z, z_{1}\right)=\frac{\left(z-z_{1}\right)\left(z-\bar{z}_{1}\right)}{\left(1-z_{1}\right)\left(1-\bar{z}_{1}\right)}, \quad z_{1}=\rho e^{i \varphi},
$$

и корень $z_{1}$ расположен в секторе

$$
\rho>0, \quad \frac{\pi}{2^{k}} \leqslant \varphi<\frac{\pi}{2^{k-1}}, \quad k \geqslant 2 .
$$

Тогда

$$
h\left(z, z_{1}\right) h\left(z,-z_{1}\right) h\left(z^{2},-z_{1}^{2}\right) \ldots h\left(z^{2^{k-2}},-z_{1}^{2^{k-2}}\right)=h\left(z^{2^{k-1}}, z_{1}^{2^{k-1}}\right) .
$$

Соотношение (10) доказывается индукцией по $k$.

Поясним смысл этой леммы. При $z=1$ все сомножители в левой части (10) и его правая часть равны 1 . Если выполнено условие леммы, то числа $z_{1}, z_{1}^{2}, \ldots, z_{1}^{2^{k-2}}$ лежат в правой полуплоскости, а числа $-z_{1},-z_{1}^{2}, \ldots,-z_{1}^{2^{k-2}}$ и $z_{1}^{2^{k-1}}-$ в левой полуплоскости. Поэтому все сомножители левой части соотношения (10), кроме первого, и его правая 
часть являются производящими функциями случайных величин, которые имеют вид соответственно $\kappa_{0}, 2 \kappa_{1}, \ldots, 2^{k-2} \kappa_{k-2}$ и $2^{k-1} \kappa_{k-1}$, где $\kappa_{0}, \kappa_{1}, \ldots, \kappa_{k-1} \in \mathrm{M}_{2}$.

Перейдем теперь непосредственно к построению компенсаторов.

Теорема 1. При любом $n \geqslant 3$ для любой с. в. $\xi \in \mathrm{M}_{n}$ существует такой конечный набор независимых с.в. $\eta_{1}, \eta_{2}, \ldots \in \mathrm{M}_{2}$, не зависящих от $\xi$, и числа $d_{1}, d_{2}, \ldots \in\{1,2, \ldots\}$, что распределение суммы $\xi+\sum_{k \geqslant 1} d_{k} \eta_{k}$ совпадает с распределением $\sum_{m \geqslant 1} c_{m} \xi_{m}$, где $\xi_{1}, \xi_{2}, \ldots \in \mathrm{M}_{1} \cup \mathrm{M}_{2}$ и независимы $, c_{1}, c_{2}, \ldots \in\{1,2, \ldots\}$.

Если все корни пр. $б . P(z)=\mathbf{E} z^{\xi}, \xi \in \mathrm{M}_{n}$, лежат в левой полуплоскости $\operatorname{Re} z \leqslant 0$, то распределение $\xi$ совпадает с распределением суммы независимых с. в. из $\mathrm{M}_{1} \cup \mathrm{M}_{2}$.

Доказательство. Производящая функция $P(z)=\mathbf{E} z^{\xi}$ с.в. $\xi$ как многочлен с неотрицательными коэффициентами может иметь некоторое количество $r$ действительных неположительных корней $-\alpha_{1}, \ldots,-\alpha_{r} \in(-\infty, 0]$, некоторое количество $2 s$ попарно комплексно сопряженных комплексных корней $\beta_{k}=\rho_{k} e^{i \varphi_{k}}, \bar{\beta}_{k}, k=1, \ldots, s$, расположенных в левой полуплоскости, и некоторое количество $2 t$ попарно комплексно сопряженных комплексных корней $\gamma_{l}=r_{l} e^{i \psi_{l}}, \bar{\gamma}_{l}$, $l=1, \ldots, t$, расположенных в правой полуплоскости, но не на положительной полуоси (и должны выполняться равенства $r+2 s+2 t=n$, $P(1)=1)$.

Тогда

$$
P(z)=\prod_{j=1}^{r} \frac{z+\alpha_{j}}{1+\alpha_{j}} \times \prod_{k=1}^{s} \frac{\left(z-\beta_{k}\right)\left(z-\bar{\beta}_{k}\right)}{\left(1-\beta_{k}\right)\left(1-\bar{\beta}_{k}\right)} \times \prod_{l=1}^{t} \frac{\left(z-\gamma_{l}\right)\left(z-\bar{\gamma}_{l}\right)}{\left(1-\gamma_{l}\right)\left(1-\bar{\gamma}_{l}\right)} .
$$

Первое произведение в правой части является производящей функцией суммы независимых бернуллиевских случайных величин из $\mathrm{M}_{1}$; обозначим их $\xi_{j}, j=1, \ldots, r$.

Второе произведение

$$
\prod_{k=1}^{s} \frac{\left(z-\beta_{k}\right)\left(z-\bar{\beta}_{k}\right)}{\left(1-\beta_{k}\right)\left(1-\bar{\beta}_{k}\right)}=\prod_{k=1}^{s} \frac{z^{2}-2 z \rho_{k} \cos \varphi_{k}+\rho_{k}^{2}}{1-2 \rho_{k} \cos \varphi_{k}+\rho_{k}^{2}}
$$

есть производящая функция суммы независимых с. в. из $\mathrm{M}_{2}$, поскольку в каждом трехчлене все коэффициенты неотрицательны. Соответствующие независимые (и не зависящие от $\xi_{1}, \ldots, \xi_{r}$ ) случайные величины обозначим $\xi_{r+1}, \ldots, \xi_{r+s}$. 
Если в (11) третье произведение отсутствует (в правой полуплоскости корней нет), то получаем доказательство второго утверждения теоремы: распределение $\xi$ совпадает с распределением

$$
\sum_{j=1}^{r} \xi_{j}+\sum_{k=1}^{s} \eta_{k}
$$

Если $P(z)$ имеет корни в правой полуплоскости (так что $t>0$ ), то третье произведение в правой части (11) имеет вид

$$
\prod_{l=1}^{t} \frac{\left(z-\gamma_{l}\right)\left(z-\bar{\gamma}_{l}\right)}{\left(1-\gamma_{l}\right)\left(1-\bar{\gamma}_{l}\right)}=\prod_{l=1}^{t} h\left(z, \gamma_{l}\right)
$$

где $h(z, \gamma)$ - та же функция, что в лемме 7, и $\gamma_{l}=r_{l} e^{i \psi_{l}}, l=1, \ldots, t$. Так как

$$
\frac{\pi}{2^{k_{l}}} \leqslant \psi_{l}<\frac{\pi}{2^{k_{l}-1}}, \quad k_{l}=\min \left\{k \in \mathbb{Z}: k \geqslant \log _{2}\left(\pi / \psi_{l}\right)\right\},
$$

то согласно лемме 7

$$
\prod_{l=1}^{t}\left(h\left(z, \gamma_{l}\right) \prod_{m=0}^{k_{l}-2} h\left(z^{2^{m}},-\gamma_{l}^{2^{m}}\right)\right)=\prod_{l=1}^{t} h\left(z^{2^{k_{l}-1}}, \gamma_{l}^{2^{k_{l}-1}}\right),
$$

и $l$-й сомножитель в правой части является производящей функцией с. в. $2^{k_{l}-1} \xi_{r+s+l}$, где $\xi_{r+s+l} \in \mathrm{M}_{2}$. С другой стороны, если $l \in$ $\{1, \ldots, t\}, m \in\left\{0,1, \ldots, k_{l}-2\right\}$, то $h\left(z^{2^{m}},-\gamma_{l}^{2^{m}}\right)$ является производящей функцией с. в. $2^{m} \eta_{l, m}, \eta_{l, m} \in \mathrm{M}_{2}$.

Из сделанных замечаний, (11) и (12) следует, что

$$
\begin{gathered}
P(z) \prod_{l=1}^{t} \prod_{m=0}^{k_{l}-2} h\left(z^{2^{m}},-\gamma_{l}^{2^{m}}\right) \\
=\prod_{j=1}^{r} \frac{z+\alpha_{j}}{1+\alpha_{j}} \times \prod_{k=1}^{s} \frac{\left(z-\beta_{k}\right)\left(z-\bar{\beta}_{k}\right)}{\left(1-\beta_{k}\right)\left(1-\bar{\beta}_{k}\right)} \times \prod_{l=0}^{t} h\left(z^{2^{k_{l}-1}},-\gamma_{l}^{2^{k_{l}-1}}\right),
\end{gathered}
$$

или

$$
\mathbf{E} z^{\xi} \cdot \prod_{l=1}^{t} \prod_{m=0}^{k_{l}-2} \mathbf{E} z^{2^{m}} \eta_{l, m}=\prod_{j=1}^{r} \mathbf{E} z^{\xi_{j}} \cdot \prod_{k=1}^{s} \mathbf{E} z^{\xi_{r+k}} \cdot \prod_{l=1}^{t} \mathbf{E} z^{2^{k_{l}-1} \xi_{r+s+l}}
$$


т. е. если $\xi, \eta_{l, m}\left(l \in\{1, \ldots, t\}, m \in\left\{0, \ldots, k_{l}-2\right\}\right)$, независимы и $\xi_{j}, j \in$ $\{1, \ldots, r+s+t\}$, независимы, то распределения

$$
\xi+\sum_{l=1}^{t} \sum_{m=0}^{k_{l}-2} 2^{m} \eta_{l, m} \quad \text { и } \sum_{j=1}^{r+s} \xi_{j}+\sum_{l=1}^{t} 2^{k_{l}-1} \xi_{r+s+l}
$$

совпадают, что и требовалось доказать.

Отметим, что если все корни производящей функции (1) лежат на мнимой оси, т. е. когда $n$ четно и

$$
P(z)=\prod_{k=1}^{n / 2} \frac{\left(z+i \beta_{k}\right)\left(z-i \beta_{k}\right)}{\left(1+i \beta_{k}\right)\left(1-i \beta_{k}\right)}=\prod_{k=1}^{n / 2} \frac{z^{2}+\beta_{k}^{2}}{1+\beta_{k}^{2}},
$$

TO

$$
\xi=2 \sum_{k=1}^{n / 2} \xi_{k}
$$

где все слагаемые в правой части - бернуллиевские случайные величины из $\mathrm{M}_{1}$.

Пример. Пусть

$$
\mathbf{E} z^{\xi}=P(z)=\frac{1}{5}\left(z^{4}+z^{3}+z^{2}+2\right)=\frac{1}{5}\left(z^{2}+2 z+2\right)\left(z^{2}-z+1\right) .
$$

Здесь $R(z)=\frac{1}{5}\left(z^{2}+2 z+2\right)$ есть производящая функция некоторой случайной величины $\eta$ из класса $\mathrm{M}_{2}$. Функция $Q(z)=z^{2}-z+1$ имеет корни в правой полуплоскости, а корни функции $Q(-z)=\frac{1}{3}\left(z^{2}+z+1\right)$ лежат в левой полуплоскости, и это есть производящая функция некоторой случайной величины $\zeta$ из класса $\mathrm{M}_{2}$, которая и является в данном случае искомым компенсатором. Следовательно,

$$
\mathbf{E} z^{\xi} \mathbf{E} z^{\zeta}=\frac{1}{3} P(z) Q(-z)=\frac{1}{5}\left(z^{2}+2 z+2\right) \frac{1}{3}\left(z^{4}+z^{2}+1\right)=\mathbf{E} z^{\eta} \mathbf{E} z^{2 \kappa},
$$

где $\mathbf{E} z^{2 \kappa}=\frac{1}{3}\left(z^{4}+z^{2}+1\right), \kappa \in \mathrm{M}_{2}$. Таким образом, если $\xi$ и $\zeta$ независимы и $\eta$ и $\kappa$ независимы, то распределения $\xi+\zeta$ и $\eta+2 \kappa$ совпадают.

\section{4. Предельные теоремы}

\section{1. Сходимость к закону Пуассона}

В этом разделе рассматривается задача о сходимости распределений последовательности целочисленных случайных величин $\xi_{n} \in \mathrm{M}_{n}, n=$ 
$1,2, \ldots$, к закону Пуассона в терминах корней их производящих функций $P_{n}(z)=\mathbf{E} z^{\xi_{n}}$. Корни (нули) пр. ф. $P_{n}(z)$ будем далее обозначать как в (3), снабжая их дополнительным индексом $n: z_{k}=z_{n, k}=$ $\rho_{n, k} e^{i \varphi_{n, k}}, k=1, \ldots, n$, так что

$$
P_{n}(z)=\prod_{k=1}^{n}\left(\frac{z-z_{n, k}}{1-z_{n, k}}\right)=\prod_{k=1}^{n}\left(b_{n, k}+a_{n, k} z\right),
$$

положим также

$$
\begin{aligned}
& \mu_{n}=\mathbf{E} \xi_{n}=\sum_{k=1}^{n} \frac{1}{1-z_{n, k}}=\sum_{k=1}^{n} a_{n, k}, \\
& \sigma_{n}^{2}=\mathbf{D} \xi_{n}=\sum_{k=1}^{n} \frac{-z_{n, k}}{\left(1-z_{n, k}\right)^{2}}=\sum_{k=1}^{n} a_{n, k} b_{n, k} .
\end{aligned}
$$

Поскольку мы рассматриваем случай, когда последовательность пр. ф. $\left\{P_{n}(z), n=1,2, \ldots\right\}$ сходится к целой функции $P(z)=e^{\lambda(z-1)}$ равномерно в любой ограниченной области плоскости $z$, а последняя функция не имеет нулей, то согласно теореме Гурвица (см., например, [7, с. 317]) все нули $z_{\eta, k}$ с ростом $n$ должны по модулю неограниченно возрастать.

Теорема 2. Условия

$$
\sum_{k=1}^{n} \frac{1}{1-z_{n, k}} \rightarrow \lambda \in(0, \infty), \quad \sum_{k=1}^{n} \frac{1}{\left|z_{n, k}\right|^{2}} \rightarrow 0 \quad(n \rightarrow \infty)
$$

достаточны для того, чтобы предельным законом для с. в. $\xi_{n}$ был закон Пуассона с параметром $\lambda, m$. е. чтобы

$$
\mathbf{P}\left\{\xi_{n}=k\right\} \rightarrow \frac{\lambda^{k}}{k !} e^{-\lambda}, \quad k=0,1,2, \ldots \quad(n \rightarrow \infty) .
$$

Доказательство. Запишем пр. ф. $P_{n}(z)$ в виде

$$
P_{n}(z)=\prod_{k=1}^{n}\left(1+\frac{z-1}{1-z_{n, k}}\right)
$$

Из условий теоремы следует, что все нули $z_{n, k}$ с ростом $n$ по модулю неограниченно растут. Поэтому можно рассматривать ее логарифм, $\ln P_{n}(z)$, по крайней мере, для конечных значений $z$ (имеется в виду 
главное значение логарифма). Разлагая логарифм в ряд Тейлора, получим

$$
\ln P_{n}(z)=\sum_{k=1}^{n} \ln \left(1+\frac{z-1}{1-z_{n, k}}\right)=\sum_{k=1}^{n} \frac{z-1}{1-z_{n, k}}+O\left(\sum_{k=1}^{n} \frac{|z-1|^{2}}{\left|1-z_{n, k}\right|^{2}}\right) .
$$

Но из условий (14) следует, что

$$
\ln P_{n}(z)=\lambda(z-1)+o(1)
$$

причем эта оценка равномерна по z в любой ограниченной области.

Отсюда получаем, что

$$
P_{n}(z)=e^{\lambda(z-1)}(1+o(1))
$$

Но это и означает, что распределение случайной величины $\xi_{n}$ слабо сходится к распределению Пуассона с параметром $\lambda$.

\section{2. Сходимость к нормальному закону}

Рассмотрим теперь условия сходимости распределений последовательности целочисленных случайных величин $\xi_{n} \in \mathrm{M}_{n}, n=1,2, \ldots$, к нормальному закону в терминах корней $z_{n, k}=\rho_{n, k} e^{i \varphi_{n, k}}, k=1, \ldots, n$, их производящих функций $P_{n}(z)=\mathbf{E} z^{\xi_{n}}$.

Лемма 8. Имеют место неравенства (см. (3))

$$
\sin \frac{\pi}{n} \leqslant\left|1-z_{n, k}\right| \leqslant 1+\rho_{n, k}
$$

при этом верхняя граница достигается для действительных корней $z_{n, k}=-\rho_{n, k}$, а нижняя - для двухточечного распределения:

$$
p_{0}=\frac{\left(\cos \frac{\pi}{n}\right)^{n}}{1+\left(\cos \frac{\pi}{n}\right)^{n}}, \quad p_{n}=1-p_{0}, \quad p_{k}=0, \quad k=1, \ldots, n-1 .
$$

Доказательство. Имеем

$$
\left|1-z_{n, k}\right|^{2}=1-2 \rho_{n, k} \cos \varphi_{n, k}+\rho_{n, k}^{2}
$$

при этом согласно лемме 1

$$
-1 \leqslant \cos \varphi_{n, k} \leqslant \cos \frac{\pi}{n}
$$


Отсюда видно, что верхняя граница в (15) достигается при $\varphi_{n, k}=\pi$, следовательно, $z_{n, k}$ - действительный корень. Нижняя же граница достигается, когда корень лежит на луче $\varphi_{n, k}=\frac{\pi}{n}$, и треугольник с вершинами $\left\{0,1, z_{n, k}\right\}$ имеет прямой угол в вершине $z_{n, k}-$ в этом случае искомый катет равен синусу противолежащего угла, поскольку гипотенуза равна единице. По лемме 2 этот корень однозначно определяет распределение из класса $\mathrm{M}_{n}$ с указанными в формулировке теоремы параметрами.

Замечание. Из (15) следует, что с ростом параметра $n$ приближение корней $z_{n, k}$ к 1 происходит по порядку не быстрее $\frac{1}{n}$.

Введем величину (см. (13))

$$
\gamma_{n}=\sigma_{n} \min _{k}\left|1-z_{n, k}\right|
$$

Укажем достаточные условия асимптотической нормальности $\xi_{n}$ при $n \rightarrow \infty$.

Теорема 3. Пусть при $n \rightarrow \infty$ выполняются условия

1) $\mathbf{D} \xi_{n}=\sigma_{n}^{2}=\sum_{k=1}^{n} \frac{-z_{n, k}}{\left(1-z_{n, k}\right)^{2}} \rightarrow \infty$,

2) $\frac{\mu_{n}}{\sigma_{n}^{3}} \rightarrow 0, \mu_{n}=\mathbf{E} \xi_{n}=\sum_{k=1}^{n} \frac{1}{1-z_{n, k}}$,

3) $\gamma_{n} \rightarrow \infty$

4) $\frac{1}{\sigma_{n}^{3}} \sum_{k=1}^{n} \frac{1}{\left|1-z_{n, k}\right|^{3}} \rightarrow 0$.

Тогда нормированная случайная величина $\tilde{\xi}_{n}=\left(\xi_{n}-\mu_{n}\right) / \sigma_{n}$ имеет в пределе стандартное нормальное распределение $\mathcal{N}(0,1)$.

Доказательство. Для характеристической функции величины $\tilde{\xi}_{n}$ имеем представление (см. доказательство теоремы 2)

$$
\mathbf{E} e^{i t \tilde{\xi}_{n}}=e^{-i t \mu_{n} / \sigma_{n}} P_{n}\left(e^{i t / \sigma_{n}}\right)=e^{-i t \mu_{n} / \sigma_{n}} \prod_{k=1}^{n}\left(1+\frac{e^{i t / \sigma_{n}}-1}{1-z_{n, k}}\right) .
$$

Отсюда и из того, что $\max _{k}\left|\frac{e^{i t / \sigma_{n}}}{1-z_{n, k}}\right| \rightarrow 0$ при $\gamma_{n} \rightarrow \infty$, следует асимпто2020, T. 11, № 1 , C. $27-46$ 
тическое разложение

$$
\begin{gathered}
\ln \mathbf{E} e^{i t \tilde{\xi}_{n}}=-i t \frac{\mu_{n}}{\sigma_{n}}+\sum_{k=1}^{n} \ln \left(1+\frac{e^{i t / \sigma_{n}}-1}{1-z_{n, k}}\right) \\
=-i t \frac{\mu_{n}}{\sigma_{n}}+\left(e^{i t / \sigma_{n}-1}\right) \sum_{k=1}^{n} \frac{1}{1-z_{n, k}} \\
-\frac{1}{2}\left(e^{i t / \sigma_{n}}-1\right)^{2} \sum_{k=1}^{n} \frac{1}{\left(1-z_{n, k}\right)^{2}}+O\left(\left(e^{i t / \sigma_{n}}-1\right)^{3} \sum_{k=1}^{n} \frac{1}{\left(1-z_{n, k}\right)^{3}}\right) .
\end{gathered}
$$

Tak кak

$$
\sum_{k=1}^{n} \frac{1}{\left(1-z_{n, k}\right)^{2}}=\sum_{k=1}^{n}\left(\frac{1}{1-z_{n, k}}-\frac{-z_{n, k}}{\left(1-z_{n, k}\right)^{2}}\right)=\mu_{n}-\sigma_{n}^{2},
$$

то, используя формулу Тейлора для $e^{u}, u \rightarrow 0$, получаем

$$
\ln \mathbf{E} e^{i t \tilde{\xi}_{n}}=-\frac{t^{2}}{2}+O\left(\frac{1}{\sigma_{n}}+\frac{\mu_{n}}{\sigma_{n}^{3}}+\frac{1}{\sigma_{n}^{3}} \sum_{k=1}^{n} \frac{1}{\left|1-z_{n, k}\right|^{3}}\right) .
$$

Все слагаемые под знаком $O$ по условиям 1, 2 и 4 теоремы стремятся к нулю, таким образом, в условиях теоремы $\ln \mathbf{E} e^{i t \xi_{n}} \rightarrow-\frac{t^{2}}{2}$, что ее и доказывает.

Отметим, что недавно в [13] доказана сходимость к нормальному закону при условиях $\sigma_{n}>n^{\varepsilon}, \gamma_{n}>\sigma_{n}^{\varepsilon}, \varepsilon>0$.

\section{5. Примеры}

Приведем ряд примеров производящих функций (1), для которых известны их корни.

- Число успехов в $n$ испьтаниях Бернулли. Для с. в. $\mu_{n}$ - числа успехов в $n$ испытаниях Бернулли с вероятностью успеха $p$ в отдельном испытании ее пр. ф. $P(x)=(q+x p)^{n}$ имеет корень кратности $n$ в точке $x=-q / p$.

- Триномиальное распределение. Так мы называем распределение с производящей функцией

$$
P(z)=\left(p_{0}+p_{1} z+p_{2} z^{2}\right)^{n},
$$

т. е. распределение суммы $n$ независимых одинаково распределенных случайных величин из класса $\mathrm{M}_{2}$. Аналогичное распределение суммы $n$ независимых неодинаково распределенных случайных величин 
из класса $\mathrm{M}_{2}$ назовем обобщенным триномиальным распределением. Его производящая функция имеет вид

$$
P(z)=\prod_{k=1}^{n}\left(p_{k 0}+p_{k 1} z+p_{k 2} z^{2}\right) .
$$

Все корни таких производящих функций лежат в левой полуплоскости.

- Случайные $r$-подстановки ([6]). Рассмотрим перестановки элементов множества $X_{n+r}=\{1,2, \ldots, r, \ldots, n+r\}, n \geqslant 1, r \geqslant 0$, имеющие не менее $r$ циклов, при дополнительном условии, что элементы $1,2, \ldots, r$ принадлежат разным циклам. В этом случае говорят об $r$ перестановках (подстановках). Число $r$-подстановок с $r+k$ циклами есть $r$-число Стирлинга первого рода $s(n, k, r)$, определяемое разложением [9]

$$
[x+r]_{n}=\sum_{k=0}^{n} s(n, k, r) x^{k},
$$

где $[x]_{n}=x(x+1) \ldots(x+n-1), n \geqslant 1,[x]_{0}=1$.

Пусть теперь на множестве всех $r$-подстановок задана вероятностная мера, согласно которой любой подстановке с $r+k$ циклами приписывается вероятность, пропорциональная $\theta^{k}$, где $\theta>0-$ произвольный параметр. Обозначим через $r+\xi_{n, r}$ общее число циклов случайной $r$-подстановки в такой параметрической модели. Тогда распределение случайной величины $\xi_{n, r}$ можно записать в виде

$$
\mathbf{P}\left\{\xi_{n, r}=k\right\}=\frac{\theta^{k} s(n, k, r)}{[\theta+r]_{n}}, \quad k=0,1,2, \ldots, n .
$$

Из (17) следует, что производящая функция случайной величины $\xi_{n, r}$ равна

$$
\mathbf{E} z^{\xi_{n, r}}=\frac{[\theta z+r]_{n}}{[\theta+r]_{n}}=\prod_{j=0}^{n-1}\left(q_{j}(r, \theta)+p_{j}(r, \theta) z\right),
$$

где $p_{j}(r, \theta)=1-q_{j}(r, \theta)=\frac{\theta}{\theta+r+j}$.

Таким образом, в данном случае корни производящей функции есть

$$
-\frac{q_{j}(r, \theta)}{p_{j}(r, \theta)}=-\frac{r+j}{\theta}, \quad j=0,1, \ldots, n-1 .
$$

Случай $r=0$ соответствует обычным (без ограничений) подстановкам, а если, дополнительно, параметр $\theta=1$, то имеем классическую 
модель равновероятных подстановок, для которой представление (18) известно еще со времен классической работы Гончарова [2].

- Рекорды в случайных перестановках. Рассмотрим n-перестановку $s=\left(s_{1}, s_{2}, \ldots, s_{n}\right)$. Говорят, что элемент $s_{j}$ является в ней рекордом, если $s_{i}<s_{j}$ для всех $i<j$, а число $s_{j}$ называют рекордным значением. Реньи (1962) показал, что число $n$-перестановок с $k$ рекордами равно их числу с $k$ циклами, т. е. равно абсолютной величине $|s(n, k)|$ числа Стирлинга первого рода, поэтому изучение числа рекордов в случайной перестановке эквивалентно изучению числа циклов в ней (см. предыдущий пример). Новым здесь является рассмотрение такой характеристики, как сумма позиций всех рекордов в перестановке.

Пусть $N(n, k)$ обозначает число тех $n$-перестановок, в каждой из которых сумма позиций ее рекордов равна $k$. Эти числа определяются разложением [15]

$$
z\left(z^{2}+1\right)\left(z^{3}+2\right) \ldots\left(z^{n}+n-1\right)=\sum_{k=0}^{n} N(n, k) z^{k} .
$$

Следовательно, производящая функция случайной величины $\xi_{n}-$ суммы позиций рекордов в случайной равновероятной перестановке - есть

$$
\mathbf{E} z^{\xi_{n}}=\frac{1}{n !} \prod_{j=1}^{n}\left(z^{j}+j-1\right)
$$

т. е. в данном случае величина $\xi_{n}$ представима в виде линейной комбинации независимых бернуллиевских случайных величин.

Нули этой функции, за исключением нуля $z=0$, лежат на концентрических окружностях радиусов $(j-1)^{1 / j}, j=2,3, \ldots, n$ и, как нетрудно убедиться, условие асимптотической нормальности для этой величины не выполняется.

- Случайные $r$-разбиения ([6]). Рассмотрим такие разбиения конечного множества $X$ на блоки, что несколько выделенных элементов $X$ должны принадлежать разным блокам. Пусть множество $X_{n+r}=$ $\{1,2, \ldots, r, \ldots, n+r\}, n \geqslant 1, r \geqslant 0$, разбивается на $r$ или более блоков так, что элементы $1,2, \ldots, r$ принадлежат разным блокам. Такие разбиения называют $r$-разбиениями, а число разбиений, имеющих $r+k$ блоков, есть $r$-число Стирлинга второго рода $S(n, k, r)$, определяемое разложением [9]

$$
(x+r)^{n}=\sum_{k=0}^{n} S(n, k, r)(x)_{k}
$$


где $(x)_{n}=x(x-1) \ldots(x-n+1), n \geqslant 1,(x)_{0}=1$.

Пусть на множестве всех таких $r$-разбиений множества $X_{n+r}$ задана вероятностная мера, согласно которой любому разбиению с $r+k$ блоками приписывается вероятность, пропорциональная $\theta^{k}$, где $\theta>0-$ произвольный параметр. Обозначим через $r+\xi_{n, r}$ общее число блоков случайного $r$-разбиения множества $X_{n+r}$ в такой параметрической модели. Тогда распределение случайной величины $\xi_{n, r}$ можно записать в виде

$$
\mathbf{P}\left\{\xi_{n, r}=k\right\}=\frac{\theta^{k} S(n, k, r)}{B_{n, r}(\theta)}, \quad k=0,1,2, \ldots, n ;
$$

нормирующий множитель

$$
B_{n, r}(\theta)=\sum_{k=0}^{n} S(n, k, r) \theta^{k}
$$

называют $r$-полиномом Белла.

Из (19) и (20) следует, что производящая функция случайной величины $\xi_{n, r}$ равна отношению $r$-полиномов Белла:

$$
\mathbf{E} z^{\xi_{n, r}}=P_{n, r, \theta}(z)=\frac{B_{n, r}(z \theta)}{B_{n, r}(\theta)} .
$$

Известно [12], что все корни полинома $B_{n, r}(\theta)$ различны, действительны и неположительны. Поэтому, обозначив его ненулевые корни через $-\alpha_{1},-\alpha_{2}, \ldots,-\alpha_{n-1}$ (явный вид этих корней неизвестен), можно записать представление

$$
B_{n, r}(\theta)=\theta\left(\theta+\alpha_{1}\right) \ldots\left(\theta+\alpha_{n-1}\right) .
$$

Отсюда следует, что

$$
P_{n, r, \theta}(z)=z \prod_{j=1}^{n-1}\left(\frac{z \theta}{\theta+\alpha_{j}}+\frac{\alpha_{j}}{\theta+\alpha_{j}}\right) .
$$

Но это означает, что случайная величина $\xi_{n, r}$ может быть представлена в виде суммы независимых бернуллиевских случайных величин:

$$
\xi_{n, r}=\xi_{n, r, 1}+\xi_{n, r, 2}+\ldots+\xi_{n, r, n-1}+1,
$$

где

$p_{n, r, j}(\theta)=\mathbf{P}\left\{\xi_{n, r, j}=1\right\}=1-\mathbf{P}\left\{\xi_{n, r, j}=0\right\}=\frac{\theta}{\theta+\alpha_{j}}, \quad j=1,2, \ldots, n-1$. 
Случай $r=0$ соответствует обычным (без ограничений) разбиениям, а если, дополнительно, параметр $\theta=1$, то мы имеем классическую модель равновероятных разбиений, для которой представление (23) впервые было получено в [11].

- Нелинейные коды ([10]). Пусть $a_{n}-n$-мерный вектор с элементами из поля характеристики $2, s-$ подстановка степени $n$ с циклами длин $n_{1}, n_{2}, \ldots, n_{k}, n_{j} \geqslant 2, j=1,2, \ldots, k, \sum_{j} n_{j}=n$. Пусть, далее, $a_{n} s-$ вектор, полученный из $a_{n}$ перестановкой его элементов по подстановке $s$, $\left\|a_{n}\right\|$ - вес Хемминга вектора $a_{n}$. Если $a_{n}$ выбирается случайно и равновероятно из множества всех двоичных $n$-мерных векторов, то сумма $\xi_{n}=\left\|a_{n} \oplus a_{n} s\right\|$ (символ $\oplus$ означает суммирование по $\left.\bmod 2\right)$ является целочисленной случайной величиной с производящей функцией

$$
P_{n}(z)=\prod_{j=1}^{k}\left[\left(\frac{1+z}{2}\right)^{n_{j}}+\left(\frac{1-z}{2}\right)^{n_{j}}\right] .
$$

Корни этого многочлена есть

$$
z_{j, l}=i \operatorname{tg} \frac{\pi(2 l+1)}{n_{j}}, \quad l=0,1, \ldots, n_{j}-1, \quad j=1, \ldots, k,
$$

кроме $l=\left(n_{j}-1\right) / 2$ при нечетных $n_{j}$.

Таким образом, в данном случае все корни лежат на мнимой оси, и дисперсия $\sigma_{n}^{2}=n / 4$ неограниченно возрастает с ростом $n$. Следовательно, с. в. $\xi_{n}$ асимптотически нормальна.

- Статистика критерия согласия для проверки гипотезы случайности. В различных статистических задачах исходные данные $X=$ $\left(X_{1}, \ldots, X_{n}\right)$ часто рассматривают как выборку из распределения некоторой случайнй величины $\xi$, т. е. считают компоненты $X_{i}$ вектора данных $X$ независимыми и одинаково распределенными случайными величинами. Такое предположение называют гипотезой случайности $H_{0}$. Критерий согласия для проверки этой гипотезы можно построить исходя из следующих соображений (далее предполагается, что вектор $X$ имеет непрерывное распределение).

Если гипотеза $H_{0}$ действительно имеет место, то компоненты вектора $X$ «равноправны», и поэтому данные не должны быть упорядочены ни в каком смысле. Другими словами, ситуацию, соответствующую гипотезе $H_{0}$, можно охарактеризовать как «полный хаос». При отклонениях от $H_{0}$ исходные данные имеют тот или иной порядок, имеются 
скрытые связи. Следовательно, соответствующий статистический критерий проверки этой гипотезы можно построить на основании статистик, измеряющих степень «беспорядка» исходных данных.

Одной из таких статистик является число инверсий в выборке. Эта статистика определяется следующим образом. Построим вариационный ряд $X_{(1)}<\ldots<X_{(n)}$ выборки. Говорят, что компоненты $X_{i}$ и $X_{j}$ образуют инверсию, если $i<j$, но $X_{i}$ стоит правее $X_{j}$ в вариационном ряду, т. е. наблюдению с меньшим номером соответствует большее значение. Пусть $T_{n}=T_{n}(X)$ - общее число инверсий для выборки $X$. Статистика $T_{n}$ является естественной мерой «беспорядка» среди наблюдений, и ее используют для построения критерия. Известно (см., например, [5, с. 363]), что производящая функция статистики $T_{n}$ имеет вид

$$
\mathbf{E} z^{T_{n}}=\frac{1}{n !} \prod_{r=1}^{n-1}\left(1+z+\ldots+z^{r}\right)=\frac{(1-z)^{-n}}{n !} \prod_{r=1}^{n}\left(1-z^{r}\right) .
$$

Все корни этой функции лежат, очевидно, на единичной окружности, дисперсия $T_{n}$ равна

$$
\mathrm{D} T_{n}=\frac{2 n^{3}+3 n^{2}-5 n}{72},
$$

и условия теоремы 3 выполнены.

Следовательно, статистики $T_{n}$ асимптотически нормальны (известный результат).

Отметим, что производящие функции такого же типа (с корнями на единичной окружности) имеют и другие известные статистики (Уилкоксона, Манна - Уитни и т. д.), используемые в различных задачах проверки статистических гипотез.

- Равномерное дискретное распределение (контрпример). Пусть с. в. $X \in \mathrm{M}_{n-1}$ имеет равномерное распределение:

$$
\mathbf{P}\{X=k\}=\frac{1}{n}, \quad k=0,1, \ldots, n-1 .
$$

В этом случае ее производящая функция есть

$$
\mathbf{E} z^{X}=\frac{1-z^{n}}{n(1-z)},
$$

все ее корни также лежат на единичной окружности, дисперсия равна

$$
\mathbf{D} X=\frac{n^{2}-1}{12},
$$


И

$$
\gamma_{n}=\sigma_{n}\left|1-e^{2 \pi i / n}\right|=\sin \frac{2 \pi}{n} \sqrt{\frac{n^{2}-1}{12}}=\frac{\pi}{\sqrt{3}}(1+o(1))
$$

не стремится к бесконечности.

Следовательно, условия теоремы 3 не выполнены, и очевидно, что с. в. $X$ не являются асимптотически нормальными при $n \rightarrow \infty$.

Авторы благодарны И.В. Харламову за полезные замечания.

\section{Список литературы}

[1] Ватутин В.А., Михайлов В. Г., "Предельные теоремы для числа пустых ячеек в равновероятной схеме размещения частиц комплектами", Теория вероятн. и ее примен., 27:4 (1982), 684-692.

[2] Гончаров В. Л., "Из области комбинаторики”, Изв. АН СССР, Сер. Матем., 8:1 (1944), $3-48$.

[3] Иванов В. А., Ивченко Г. И., Медведев Ю. И., “Дискретные задачи в теории вероятностей”, ВИНИТИ, Итоги науки и техники, Сер. Теория вероятн., Матем. статист., Теор. киберн., 22 (1984), 3-60.

[4] Ивченко Г.И., Медведев Ю.И., "О случайных подстановках", Труды по дискретной математике, 5 (2002), 73-92.

[5] Ивченко Г. И., Медведев Ю. И., Введение в математическую статистику, М.: Изд. ЛКИ, 2010, 600 с.

[6] Ивченко Г.И., Медведев Ю.И., "Параметрические модели случайных $r$-подстановок и $r$-разбиений и их вероятностно-статистический анализ", Математические вопросы криптографии, 9:1 (2018), 47-64.

[7] Маркушевич А.И., Теория аналитических функиий, М.-Л.: ГИТТЛ, 1950, 704 с.

[8] Сачков В.Н., Введение в комбинаторные методы дискретной математики. 2-е изд., М.: МЦНМО, 2004, 424 c.

[9] Broder A.Z., "The $r$-Stirling numbers", Discrete Math., 49:3 (1984), 241-259.

[10] McEliece R., "On the symmetry of good nonlinear codes", IEEE Trans. Inf. Theory, IT-16, 5 (1970), 609-611.

[11] Harper L., "Stirling behavior is asymptotically normal", Ann. Math. Stat, 38:2 (1961), 410-414.

[12] Mezö I., "On the maximum of r-Stirling numbers", Adv. Appl. Math., 41:3 (2008), 293-306.

[13] Michelen M., Sahasrabudhe J., "Central limit theorems from the roots of probability generating functions", 2018, arXiv: 1804.07696v2.

[14] Obrechkoff N., "Sur un problem de Laguerre", C. R. Acad. Sci., 177 (1923), 102-104.

[15] Kortchemski I., "Asymptotic behavior of permutation records", J. Comb. Theory. Ser. A, 116 (2009), 154-166. 